\title{
Impacto del sistema de cable aéreo (Metrocable) sobre la accesibilidad en la comuna \#1 de Medellín
}

\author{
Cesar Arango Gómez \\ Asistente de Proyectos, Universidad de los Andes, Colombia \\ e-mail: c.arango954@uniandes.edu.co \\ Juan Miguel Velásquez Torres \\ Instructor, Universidad de los Andes, Colombia \\ e-mail: jm.velasquez148@uniandes.edu.co \\ Juan Pablo Bocarejo Suescún \\ Profesor Asistente, Universidad de los Andes, Colombia \\ e-mail: jbocarej@uniandes.edu.co
}

Área temática: Transporte público urbano

\section{RESUMEN}

La ciudad de Medellín inauguró en el año 1995 un sistema de Metro, sin embargo en el año 2004 todavía existían algunas zonas de la ciudad con problemas de transporte. Este es el caso de la comuna \#1 de Medellín, la cual a pesar de tener la estación de Metro Acevedo a una distancia entre los $800 \mathrm{~m}$ y los $2500 \mathrm{~m}$ según el barrio, su acceso era difícil debido principalmente a factores como la topografía, bajos niveles de ingreso y la deficiente oferta de transporte público. Con el objetivo de superar dichos problemas, la alcaldía de Medellín decidió construir un cable aéreo, Metrocable, como sistema de transporte público en dicha comuna e integrarlo al Metro.

El propósito de este trabajo es establecer la variación de la accesibilidad del Metrocable en la comuna \#1 de Medellín con un índice por zona, el cual se basa en el índice de Hansen, el cual expresa la accesibilidad de una zona i (Ai) como:

$$
A_{i}=\sum_{j} a_{j} f\left(d_{i j}\right)
$$

Donde: $a_{j}$ se refiere a la atractividad de la zona que en este caso estaba medida en número de puestos de trabajo disponibles. $\mathrm{f}\left(\mathrm{d}_{\mathrm{ij}}\right)$ se refiere al costo generalizado para viajar entre la zona i y la zona j. Este costo es una función de varios parámetros.

Para el análisis se trabajó a partir de datos obtenidos de la encuesta de calidad de vida y la matriz origen destino para diferentes barrios de la comuna 1 y otros sitios representativos de la ciudad. El índice se obtiene inicialmente para el año 2000, cuando no existía el Metrocable, y posteriormente para el 2005 cuando el mismo ya estaba en operación, obteniendo aumentos en la accesibilidad entre 5 y 16 puntos porcentuales temporalmente, y mayores a 10 puntos porcentuales espacialmente. De esta forma se determina el impacto en 
la accesibilidad del proyecto.

\section{INTRODUCCIÓN}

A través de los años el concepto de accesibilidad ha sido utilizado en diferentes campos; sin embargo, en todos estos la definición de accesibilidad varía, según los puntos de vista de diferentes autores de acuerdo a sus estudios.

La accesibilidad en el trasporte según la definen (Geurs y Van Wee, 2004) "expresa la imposibilidad de una persona para cubrir la distancia entre el origen y el destino usando un modo de transporte específico; incluyendo el tiempo gastado (tiempo de viaje, espera y parqueo), costos (fijos y variables), el esfuerzo (incluyendo la confiabilidad, nivel de comodidad, riesgo de accidente, etc..)"

A pesar de que los temas de investigación en este momento, se basan en el desarrollo de metodologías de medición y evaluación de sistemas de transporte público, el tema de accesibilidad ha sido poco estudiado como indicador de calidad de transporte en el mundo.

Dado lo anterior, el propósito de este trabajo se centra en establecer la variación de la accesibilidad del Metrocable en la comuna \#1 de Medellín con un índice por zona, el cual se ha desarrollado a partir de medidas integrales de accesibilidad basadas en la ecuación de Hansen, que incluyen el tiempo promedio de viaje, porcentaje del ingreso utilizado en el transporte, y empleos disponibles, entre otros.

Adicionalmente se busca establecer la variación de la accesibilidad para la comuna \#14, El Poblado, como barrio control, el cual es de esperarse presente una accesibilidad muy superior a la de la comuna \#1, debido a que su nivel socio-económico es mucho mayor.

Con dichos resultados para ambas comunas podemos establecer el impacto que tuvo el sistema Metrocable en la accesibilidad de la Comuna \#1 de Medellín y así poder usarlo como un evaluador para el sistema.

\section{ANTECEDENTES}

\subsection{Qué es la accesibilidad}

A lo largo de los años el concepto de accesibilidad ha sido utilizado en diferentes campos; sin embargo, en todos estos la definición de accesibilidad varía, según los puntos de vista de diferentes autores de acuerdo a sus estudios, las cuales en el área de transporte han sido recopiladas por (Geurs y Van Wee, 2004) y sirven para darnos una idea general del concepto de accesibilidad. Estas definiciones son: el potencial de oportunidades de interacción (Hansen, 1959), la facilidad para alcanzar cualquier zona de actividad usando un sistema de transporte determinado (Dalvi y Martin, 1976), la libertad de los individuos para decidir si desea o no participar en diferentes actividades (Burns, 1979), y los beneficios proporcionados por un sistema de transporte y uso de la tierra dado (Ben-Akiva and Lerman, 1979), entre otros. 
Como se puede ver la accesibilidad tiene diferentes definiciones inclusive dentro de la misma área de estudio. Es por esto que (Geurs y Van Wee, 2004) hacen una explicación más general de este concepto, donde identifican 4 tipos de componentes desde los cuales puede ser explicada la accesibilidad, estos son: el uso de la tierra, el transporte, el tiempo y el individual:

1. "El componente del uso de la tierra refleja el sistema del uso de la tierra, que consiste en: a) las oportunidades de distribución de la cantidad calidad y espacio suministrado en cada destino (trabajos, tiendas, salud, y servicios sociales y de recreación, etc.), b) la demanda de estas oportunidades en los lugares de origen (donde los habitantes viven), y c) la confrontación de la oferta y demanda de oportunidades, que resultan en una competencia de actividades con capacidad restringida, como puestos de trabajo, estudio y camas de hospital.

2. El componente de transporte describe el sistema de transporte, expresado en la imposibilidad de una persona para cubrir la distancia entre el origen y el destino usando un modo de transporte especifico. Se incluye el tiempo gastado (tiempo de viaje, espera y parqueo), costos (fijos y variables), el esfuerzo (incluyendo la confiabilidad, nivel de comodidad, riesgo de accidente, etc..), la imposibilidad resulta de la confrontación ente oferta y demanda. La oferta de infraestructura incluye su ubicación y características (velocidad máxima de viaje, numero de líneas, horarios de transporte público, costo de viaje, etc.). La demanda se refiere tanto a los pasajeros como a los viajes de carga.

3. El componente del tiempo refleja las restricciones temporales, es decir, la disponibilidad de oportunidades a diferentes horas del día, y el tiempo disponible para cada individuo para participar en ciertas actividades (trabajo, recreación, etc.)

4. El componente individual refleja las necesidades (dependiendo de la edad, ingreso, nivel de educación, situación familiar, etc.), habilidades (dependiendo de la condición física de las personas, la disponibilidad de modos de transporte, etc.) y oportunidades (dependiendo del ingreso de las personas, el presupuesto de viaje, nivel de educación, etc.) de los individuos. Estas características influyen en el nivel de acceso que una persona tiene a los modos de transporte (por ejemplo, ser capaz de manejar un carro) y las oportunidades de distribución espacial (por ejemplo tienen las habilidades y el nivel de educación para acceder a puestos de trabajo calificados cerca de su área de residencia) y pueden influir enormemente en el resultado de la accesibilidad global. Varios estudios (Cervero et al., 1997; Shen, 1998; Geurs and Ritsema van Eck, 2003) han demostrado que en el caso de la accesibilidad a los trabajos, la inclusión de igualar el trabajo afecta en gran medida los indicadores de accesibilidad resultantes." (Geurs y Van Wee 2004)

\subsection{La accesibilidad como indicador}

La accesibilidad puede ser usada como indicador social o económico dependiendo del enfoque que se le quiera dar al estudio. 
“....como indicador social se utiliza para evidenciar la existencia de oportunidades económicas y sociales para las personas o grupos de personas. Es decir, el nivel de acceso a fuentes esenciales como oportunidades de trabajo, zonas de abastecimiento, salud, y servicios sociales, además de la oportunidad de interacción social con familiares $\mathrm{y}$ amigos.

Los impactos económicos generalmente se clasifican en: a) impactos directos, son aquellos costos y beneficios relacionados directamente con un proyecto, y contemplan principalmente ahorros en tiempos y costos de viaje y b) impactos indirectos, son los efectos no relacionados directamente con el proyecto, por ejemplo, mejoras en la productividad y la distribución de los ingresos.

En general, una medida de accesibilidad puede ser utilizada como indicador del beneficio económico si se encuentra relacionada directamente con la teoría económica, o puede servir como insumo para el cálculo de los impactos económicos de una intervención sobre los usos del suelo o los sistemas de transporte." (Geurs y Van Wee 2004)

\section{METODOLOGÍA}

A pesar de las múltiples formas de calcular la accesibilidad de una zona, resulta difícil cuantificarla, y no existe una mejor aproximación para medirla (Gutiérrez, 2001). Sin embargo, la mayoría se centran en la combinación de medidas del costo de viaje hacia una determinada zona, y la atractividad de la misma, expresados en términos de un mismo indicador. Ejemplos de este tipo de estudios se encuentran en Geertrman y Ritserma (1995), (Oviedo 2010).

Entre los diferentes enfoques para medir la accesibilidad, las medidas más utilizadas se basan en la combinación de oportunidades y de impedancias. Un ejemplo de este tipo de indicador es denominado modelo de gravedad, regularmente empleado por muchas instituciones de investigación (Niemeier, 1997; Bocarejo y Oviedo 2010)

\subsection{Formulación matemática del problema}

Este proyecto se realizó calculando la accesibilidad con la ecuación convencional propuesta por Hansen en 1959, la cual fue la primera medida de gravedad propuesta y la más utilizada en la literatura en los últimos años. Esta se define como:

$$
A_{i}=\sum_{j} a_{j} f\left(d_{i j}\right)
$$

Donde:

$\mathrm{Ai}=$ Accesibilidad de la zona $\mathrm{i}$ (zona de origen)

aj = Atractividad de la zona j (zona de destino), para ese caso estaba medido en número de puestos de trabajo disponibles en la zona

$\mathrm{f}(\mathrm{dij})=$ función generalizada del costo de viajar entre las zonas i y j, el cual esta dado en función de varios parámetros 
La atractividad de la zona puede medirse de varias maneras dependiendo de la disponibilidad de información, y el tipo de estudio que se quiera hacer, y como explican (Bocarejo y Oviedo, 2010), las actividades localizadas en la zona de destino son el principal indicativo, y recomiendan centrar el análisis en motivos de viaje obligatorios como estudio, trabajo o salud, donde se pueden utilizar los puestos de trabajo, cupos en instituciones educativas y disponibilidad de camas en entidades prestadoras de salud de la zona como índice de atractividad de la misma. Lo anterior debido a que la disponibilidad de información es muy limitada y resulta complicado determinar el numero total de todas las actividades de una zona. Dado el contexto y debido a la disponibilidad de información, en este estudio se utilizaron los puestos de trabajo disponibles en la ciudad de Medellín

Como muestran (Bocarejo y Oviedo, 2010), en la ecuación (1), la función de costo se define por lo general en términos del costo generalizado de viaje para movilizarse de una zona a otra y adopta la forma estándar de las funciones de utilidad, obteniendo:

$$
f\left(d_{i j}\right)=e^{-\beta C_{i j}}=e^{-\left(\beta_{1} C t+\beta_{2} C c\right)}
$$

Donde:

$\mathrm{Cij}=$ Costo generalizado de viaje entre la zona $\mathrm{i} \mathrm{y} \mathrm{j}$

$\mathrm{Ct}=$ Tiempo de viaje entre las zonas $\mathrm{i} \mathrm{y} \mathrm{j}$

$\mathrm{Cc}=$ Costo monetario del viaje

En la ecuación (2) de costo generalizado de viaje, el primer termino Ct representa el "costo" del tiempo de viajar entra las zonas i y j, variable que esta relacionada con los modos de transporte disponibles en la zona los cuales afectan los tiempos de caminata, espera, tiempo de viaje y otras variables socioeconómicas que afectan el presupuesto en tiempo de viaje que disponen los habitantes.

El segundo termino Cc representa el costo monetario del viaje, el cual esta directamente relacionado con el ingreso de los habitantes y el modo de transporte disponible en la zona

\subsection{Fuentes de información y selección de la información}

En este estudio se utilizó principalmente la información de las encuestas origen destino de 2000 y 2005 para el Área Metropolitana del Valle de Aburrá realizadas por la Universidad Nacional de Colombia sede Medellín, en donde podemos encontrar información de la movilidad urbana y una descripción detallada de los patrones de movilidad de la Región Metropolitana del Valle de Aburrá. Con estas podemos obtener las matrices de viajes entre diferentes zonas, y encontrar información socioeconómica y de hogares necesaria para la selección y clasificación de las áreas de estudio.

De la encuesta O/D también se obtuvo el número de trabajos disponibles en cada zona, como la sumatoria de los viajes con motivo trabajo desde toda la Región Metropolitana del Valle de Aburrá, a la zona i, asumiendo que un viaje de trabajo implica que en dicha zona hay un puesto de trabajo. Esta información fue validada con los resultados del censo 
general de 2005 realizado por el DANE, el cual nos da el total de unidades económicas por cada comuna y un número equivalente de puestos de trabajo por cada una de estas.

Adicionalmente se utilizó información de la Encuesta de Calidad de Vida 2005 de Medellín, realizada por el Departamento Administrativo de Planeación Nacional (DNP), en la cual se tiene información de variables demográficas, socioeconómicas y físicas a nivel de comuna en la ciudad. De esta encuesta se utilizaron los datos de población en edad de trabajar para realizar los cálculos.

\subsection{Selección de las áreas de estudio}

Se seleccionó la comuna \#1 de Medellín (imagen 1) en la cual se encuentra el primer Metrocable y es la zona donde mayor impacto tiene este proyecto. Dentro de esta comuna se seleccionaron 3 barrios, tomando en consideración principalmente la distancia de los mismos al sistema Metro y al Metrocable. De acuerdo a lo anterior se seleccionaron los barrios Granizal, Santo Domingo Savio 2 y Villa de Guadalupe.

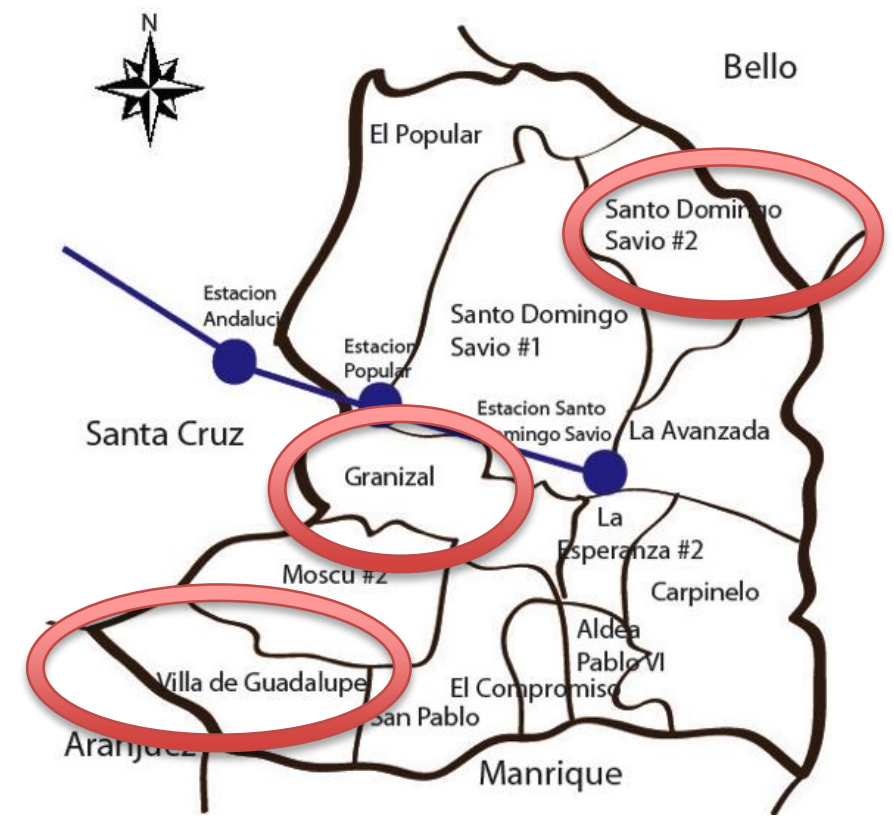

Figura 1 - Comuna \#1 Popular

Fuente: Elaboración propia a partir de mapas de http://www.medellin.gov.co/

El primer barrio seleccionado es Granizal identificado dentro de la encuesta O/D con el código 100104, el cual fue seleccionado por ser el barrio más cercano al Metrocable, esta ubicado en zona centro de la comuna contiguo a las estaciones Popular y Santo Domingo Savio del sistema Metrocable, y por su cercanía es donde se espera mayor impacto del sistema.

El segundo barrio seleccionado fue Santo Domingo Savio 2, identificado con el código 100102. Está ubicado en la zona nor-oriental de la comuna y se seleccionó ya que es uno de los más alejados con respecto a los dos sistemas de transporte, tanto el Metro como el 
Metrocable, y es de esperarse que el impacto del sistema en este barrio sea mucho menor, debido a la mayor distancia a las estaciones.

El tercer barrio seleccionado fue Villa de Guadalupe, identificado con el código 100106, ubicado en la parte nor-occidental de la comuna, el cual fue seleccionado por ser un barrio alejado del sistema Metrocable, pero cercano al sistema Metro. Se espera que el impacto del Metrocable en este barrio sea menor ya que con la estación del sistema Metro cercana, ya presenta una buena accesibilidad a los empleos de la ciudad sin la implementación del sistema Metrocable.

Adicionalmente se seleccionó la comuna \#14 de Medellín (El Poblado) (imagen 2) por ser una comuna de estrato alto y la cual tiene los mayores ingresos de la ciudad. Esto implica que tienen un buen acceso a transporte privado, adicional a los sistemas de transporte públicos los cuales también tienen una cobertura buena dentro de esta comuna.

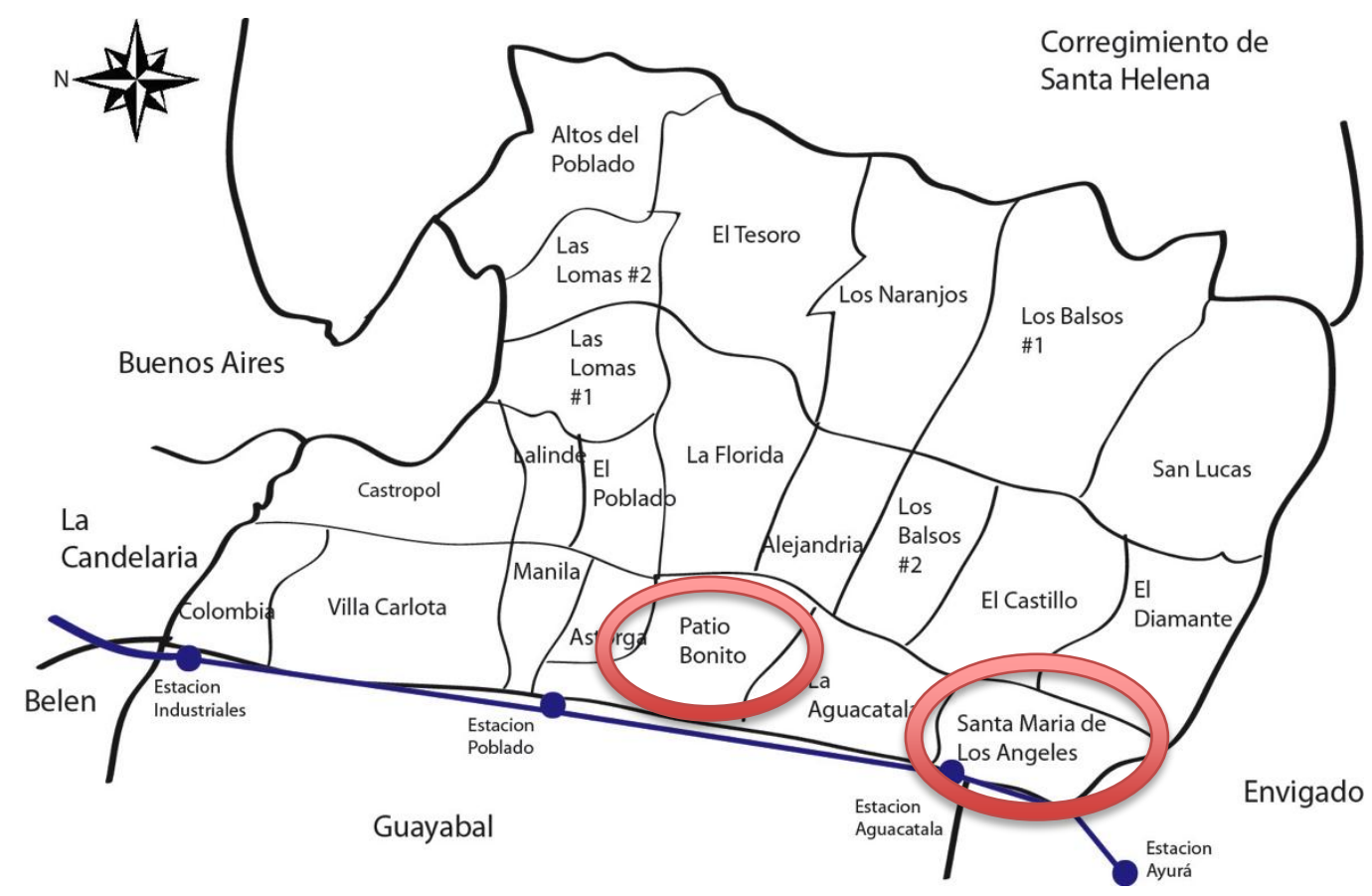

Figura 2 - Comuna \#14 El Poblado

Fuente: Elaboración propia a partir de mapas de http://www.medellin.gov.co/

La selección de estos 5 barrios con respecto a otros de similares características se hizo teniendo en cuenta el mayor número de datos dentro de la encuesta con el fin de tener una muestra estadísticamente aceptable para realizar los cálculos.

\section{RESULTADOS}

\subsection{Resultados obtenidos}

Con la información anteriormente nombrada y después de realizar las regresiones para cada barrio, con la ayuda de software especializado, se obtuvieron los factores de la ecuación 
$\mathrm{f}\left(\mathrm{d}_{\mathrm{ij}}\right)$ para cada barrio en cada año, las cuales se muestran en las siguientes ecuaciones y reflejan como responde la población de cada área frente a las variables estudiadas.

Para el año 2000 las ecuaciones de costo generalizado de viaje fueron:

$$
\begin{gathered}
f\left(d_{\text {Granizal } j}\right)=e^{(-0,03 * \mathrm{Ct}-17,87 * \mathrm{Cc})} \\
f\left(d_{\text {Santo Domingo Savio } 2 i}\right)=e^{(-0,08 * \mathrm{Ct}-10,51 * \mathrm{Cc})} \\
f\left(d_{\text {Villa de Guadalupe } i}\right)=e^{(-0,01 * \mathrm{Ct}-34,29 * \mathrm{Cc})} \\
f\left(d_{\text {Patio Bonito } i}\right)=e^{(-0,07 * \mathrm{Ct}-89,24 * \mathrm{Cc})} \\
f\left(d_{\text {Sta Maria de los Angeles } i}\right)=e^{(-0,14 * \mathrm{Ct}-25,65 * \mathrm{Cc})}
\end{gathered}
$$

para el año 2005 la ecuaciones de costo generalizado de viaje fueron:

$$
\begin{gathered}
f\left(d_{\text {Granizal } j}\right)=e^{(-0,04 * \mathrm{Ct}-18,35 * \mathrm{Cc})} \\
f\left(d_{\text {Santo Domingo Savio } 2 i}\right)=e^{(-0,06 * \mathrm{Ct}-14,95 * \mathrm{Cc})} \\
f\left(d_{\text {Villa de Guadalupe } i}\right)=e^{(-0,001 * \mathrm{Ct}-12,88 * \mathrm{Cc})} \\
f\left(d_{\text {Patio Bonito } i}\right)=e^{(-0,08 * \mathrm{Ct}-10,68 * \mathrm{Cc})} \\
f\left(d_{\text {Sta Maria de los Angeles } i}\right)=e^{(-0,14 * \mathrm{Ct}-79,23 * \mathrm{Cc})}
\end{gathered}
$$

\begin{tabular}{|c|c|c|c|c|c|}
\hline & & & 2005 & & \\
\hline & & Comuna & & Con & 14 \\
\hline Nombre Barrio & Granizal & $\begin{array}{c}\text { Sto. } \\
\text { Domingo } \\
\text { Savio } 2\end{array}$ & $\begin{array}{c}\text { Villa } \\
\text { Guadalupe }\end{array}$ & $\begin{array}{l}\text { Patio } \\
\text { Bonito }\end{array}$ & $\begin{array}{c}\text { Sta. } \\
\text { María } \\
\text { de los }\end{array}$ \\
\hline
\end{tabular}

Con estas ecuaciones de costo generalizado de viaje para cada barrio, se calculó la accesibilidad de cada uno con la ecuación (1), y los resultados obtenidos se resumen en la tabla 1 para el año 2000 y en la tabla 2 para el año 2005. En el 2000 se omitió el barrio Villa Guadalupe por falta de datos estadísticamente representativos en la encuesta O/D de 2000 para este.

\begin{tabular}{|l|c|c|c|c|}
\cline { 2 - 5 } \multicolumn{1}{c|}{} & \multicolumn{4}{c|}{2000} \\
\cline { 2 - 5 } \multicolumn{1}{c|}{} & \multicolumn{2}{c|}{ Comuna 1 } & \multicolumn{2}{c|}{ Comuna 14 } \\
\hline Nombre Barrio & Granizal & $\begin{array}{c}\text { Villa } \\
\text { Guadalupe }\end{array}$ & $\begin{array}{c}\text { Patio } \\
\text { Bonito }\end{array}$ & $\begin{array}{c}\text { Sta María de } \\
\text { los Ángeles }\end{array}$ \\
\hline Código barrio & 101004 & 100106 & 101421 & 101423 \\
\hline Total empleos Medellín & \multicolumn{4}{c|}{534.863} \\
\hline Total empleos accedidos & 4.105 & 4.620 & 4.812 & 8.057 \\
\hline Población en edad de trabajar & 9.061 & 10.467 & 4.727 & 7.958 \\
\hline $\begin{array}{l}\text { \% de la población en edad de } \\
\text { trabajar con acceso a empleo }\end{array}$ & $45 \%$ & $44 \%$ & $102 \%$ & $101 \%$ \\
\hline$\beta 1$ & $-0,03$ & $-0,01$ & $-0,07$ & $-0,14$ \\
\hline$\beta 2$ & $-17,87$ & $-34,29$ & $-89,24$ & 25,65 \\
\hline $\mathrm{t} \beta 1$ & $-5,18$ & $-3,09$ & $-1,42$ & $-5,97$ \\
\hline $\mathrm{t} \beta 2$ & $-6,76$ & $-12,11$ & $-0,99$ & 0,86 \\
\hline
\end{tabular}

Tabla 1 - Resumen resultados 2000 


\begin{tabular}{|l|c|c|c|c|c|}
\hline & & \multicolumn{3}{|c|}{} & \multicolumn{2}{c|}{ Ángeles } \\
\hline Código barrio & 100104 & 100102 & 100106 & 101421 & 101423 \\
\hline Total empleos Medellín & \multicolumn{4}{|c|}{549.904} \\
\hline Total empleos accedidos & 4.531 & 1.351 & 6.293 & 8.383 & 5.818 \\
\hline $\begin{array}{l}\text { Población en edad de } \\
\text { trabajar }\end{array}$ & 9.115 & 4.192 & 10.531 & 8.006 & 5.694 \\
\hline $\begin{array}{l}\text { \% de la Población en edad } \\
\text { de trabajar con acceso a } \\
\text { empleo }\end{array}$ & $50 \%$ & $32 \%$ & $60 \%$ & $105 \%$ & $102 \%$ \\
\hline$\beta 1$ & $-0,04$ & $-0,06$ & 0,001 & $-0,08$ & $-0,02$ \\
\hline$\beta 2$ & $-18,35$ & $-14,95$ & $-12,68$ & $-10,88$ & $-79,23$ \\
\hline $\mathrm{t} \beta 1$ & $-4,05$ & $-0,78$ & $-1,36$ & $-8,40$ & $-3,75$ \\
\hline $\mathrm{t} \beta 2$ & $-4,01$ & $-0,64$ & $-10,10$ & $-1,53$ & $-4,55$ \\
\hline
\end{tabular}

Tabla 2 - Resumen resultados 2005

\subsection{Análisis e interpretación de los resultados obtenidos}

Los resultados obtenidos para cada barrio evidencian que las condiciones geográficas, socioeconómicas y de transporte impactan de una manera significativa en el acceso a los empleos.

Realizando un análisis espacial podemos ver grandes diferencias en la accesibilidad, no solo entre las comunas 1 (figura 3 ) y 14 (figura 4) sino también entre los barrios de las mismas.

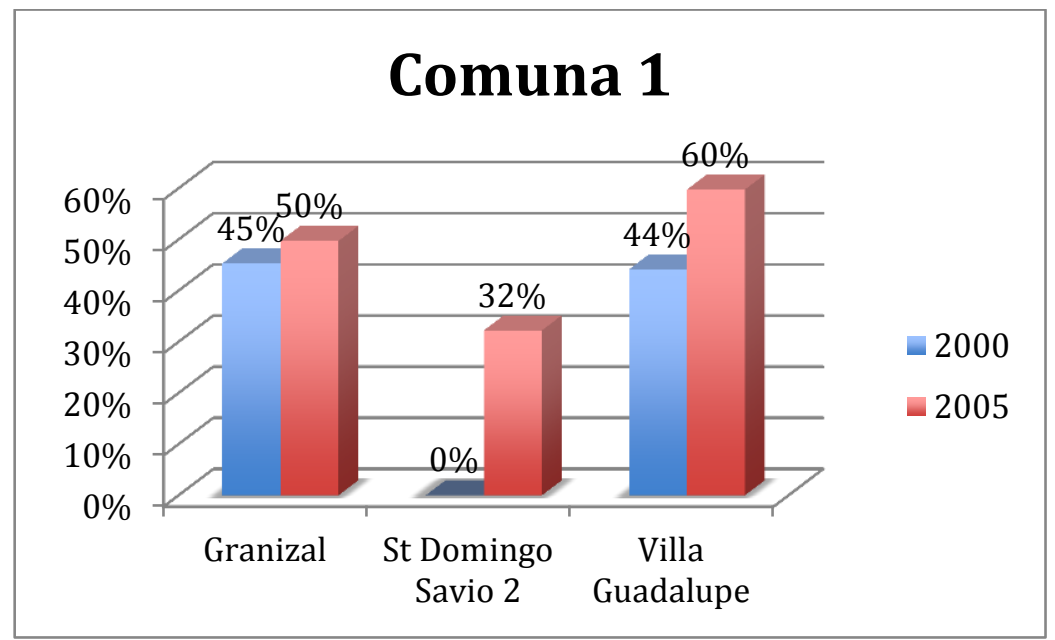

Figura 3 - Accesibilidad comuna \#1 


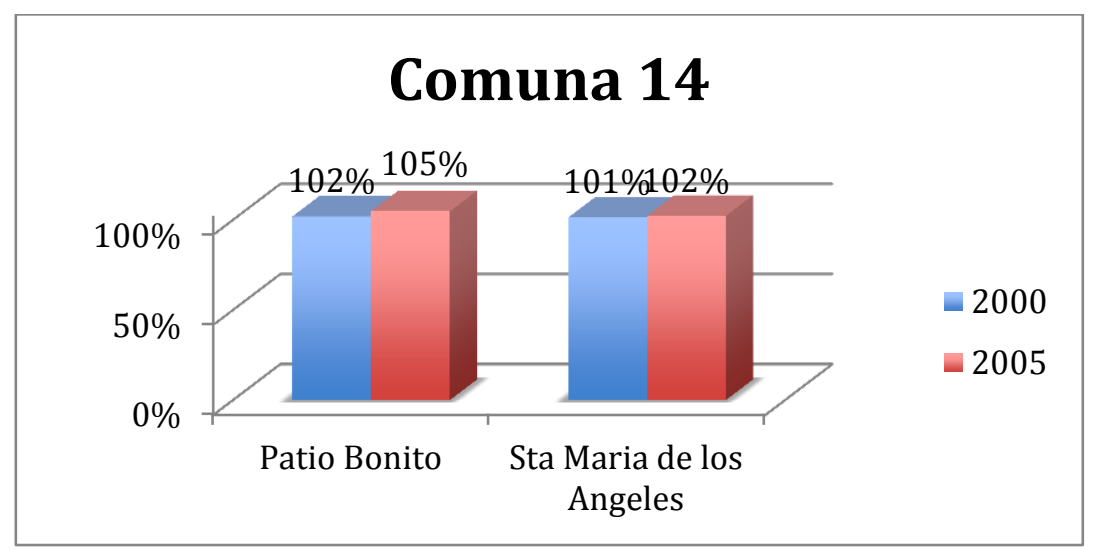

Figura 4 - Accesibilidad comuna \#14

En el caso de la comuna 1 se puede observar como el barrio Villa de Guadalupe, ubicado en la parte nor-occidental de la comuna (parte baja), presenta una accesibilidad del $60 \%$ siendo la más alta de los tres barrios estudiados. Esto puede ser debido a su ubicación cercana al Metro de Medellín, lo cual reduce el tiempo de viaje y el porcentaje de ingreso empleado en el transporte, lo cual incrementa la posibilidad de la población para acceder a los empleos de la ciudad.

El barrio Granizal, ubicado en la parte centro de la comuna contiguo a las estaciones Popular y Santo Domingo Savio del sistema Metrocable, presenta una accesibilidad del $50 \%$, en este caso la reducción en la accesibilidad con respecto al barrio Villa Guadalupe se puede explicar por el tiempo promedio de viaje el cual se ve incrementado por el tiempo de viaje en el sistema Metrocable.

En el tercer barrio estudiado, Santo Domingo Savio \#2, se obtuvo una accesibilidad del $32 \%$, este resultado tan bajo es debido a la ubicación del barrio el cual se encuentra en la zona nor-oriental de la comuna 1, alejado tanto del sistema Metrocable y del sistema Metro, por lo tanto el tiempo de viaje promedio es el más alto de los 3 barrios y el porcentaje del ingreso empleado en el transporte también es más alto con respecto a los otros barrios de la comuna, lo que genera una reducción significativa en la accesibilidad.

En la comuna 14, El Poblado, en los barrios estudiados, los cuales fueron Patio Bonito y Santa María de los Ángeles, se obtuvieron accesibilidades de $105 \%$ y $102 \%$ esto debido a que pertenecen a estratos socio-económicos altos y el porcentaje del ingreso empleado en el transporte es muy bajo con respecto a los estratos bajos. Adicionalmente a los tiempos de viajes mas cortos, ya que la mayoría de la población tiene vehículo particular y la amplia oferta de transporte que hay en esta zona de la ciudad.

Para poder realizar un análisis temporal, se calculó la accesibilidad para los mismos barrios en el año 2000, en la cual se obtuvo que para la comuna 14, la accesibilidad no obtuvo un cambio significativo, pues entre 2000 y 2005 fue del 3 puntos porcentuales para Patio Bonito y de 1 punto porcentual para Santa María de los Ángeles.

Para la comuna 1 sí se presentan grandes cambios en la accesibilidad, en el barrio 100104 
Granizal, es el barrio con mayor impacto del Metrocable. Se obtuvo una accesibilidad del $45 \%$, lo que significa que con la implementación del Metrocable, se incrementó la accesibilidad en 5 puntos porcentuales debido a la reducción del tiempo promedio de viaje y porcentaje del ingreso empleado en el transporte.

En el barrio Villa Guadalupe, se obtuvo un incremento en la accesibilidad del año 2000 al 2005 de 16 puntos porcentuales, este es el barrio con mayor incremento en la accesibilidad y puede deberse a la cercanía que tiene con los 2 sistemas de transporte Metro y Metrocable lo que genera menores tiempos de viaje y menor porcentaje del ingreso empleado en el transporte del año 2005 con respecto al año 2000.

En el barrio Santo Domingo Savio \#2 no se pudo establecer la variación en la accesibilidad debido a que para el año 2000 no se encontraban datos estadísticamente representativos para poder realizar el calculo de la accesibilidad.

Por otro lado, pero no menos importante, el resultado obtenido a partir de los coeficientes de la función de costo generalizado, ecuación (2), las cuales se muestran para cada barrio con sus respectivos coeficientes en las ecuaciones (3) a (12) y en la tabla 1 donde se observa que para todos los barrios estudiados, tanto de la comuna 1 y de la comuna 14, la variable que domina la función, es la del costo monetario del viaje Cc. Lo que no era de esperarse debido a los resultados obtenidos por (Bocarejo y Oviedo 2010) los cuales dan que para la ciudad de Bogotá en los estratos socio-económicos altos la variable que dominaba la ecuación era el tiempo de viaje y no el costo monetario.

Lo anterior se puede explicar debido a que para la Región Metropolitana del Valle de Aburrá, el tiempo promedio de viaje es de 25 minutos según la encuesta O/D de 2005. Esto implica que para la mayoría de los habitantes de la región, un incremento moderado en el tiempo de viaje no sea de mucha importancia debido a los bajos tiempos que se tienen actualmente.

\section{CONCLUSIONES}

Según los resultados obtenidos, podemos observar que el Metrocable sí tuvo un impacto significativo en la accesibilidad de la comuna 1. Este impacto se dio principalmente en el barrio Villa de Guadalupe, en donde se obtuvo un aumento del 16 puntos porcentuales que se puede explicar por la cercanía a los 2 sistemas Metro y Metrocable.

En el barrio Granizal se obtuvo una mejora en la accesibilidad del 5 puntos porcentuales lo cual es un incremento importante y en principio se le puede atribuir directamente al Metrocable ya que es el principal sistema de transporte de este barrio y el Metro queda a una gran distancia.

La comuna 14, tiene en los dos barrios estudiados una accesibilidad mayor al 100\%, lo cual se explica por la capacidad económica de sus habitantes, y por la amplia cobertura de sistemas de transporte en esta zona, y el alto número de vehículos privados, lo cual genera una mayor facilidad para movilizarse y acceder a los empleos de la ciudad. 
Adicionalmente se puede ver en los coeficientes de la función de costo generalizado como en la ciudad de Medellín, a las 2 comunas estudiadas, con sus amplias diferencias socioeconómicas, la variable que domina la ecuación es el costo del viaje, esto puede ser debido al poco tiempo promedio de viaje que se registra en todo el Valle de Aburra y que un incremento moderado en este no le afectaría a la población.

\section{BIBLIOGRAFÍA}

BEN-AKIVA, M. L. (1979). Disaggregate travel and mobility choice models and measures of accessibility. In: Hensher, D.A., Sopher, P.R. (Eds.). Behaviournal Travel Modelling. Croom Helm, Andover, Hans , 654-679.

BOCAREJO, J. P., \& OVIEDO, D. (2010). Transport accessibility and social exclusion: a better way to evaluate public transport investment? World Conference on Transport Research Society, n/d.

BURNS, L. (1979). Transportation, Temporal and Spatial Components of Accessibility. Lexington/Toronto: Lexington Books.

CERVERO, R. (2005). Accessible Cities and Regions: A Framework for Sustainable Transport and Urbanism in the 21 st Century . n/d.

CERVERO, R. (1997). Job accessibility as a performance indicator: An analysis of trends and their social policy implications in the San Francisco Bay Area. Berkely: University of California.

DALVI, M., \& MARTIN, K.M. (1976). The measurement of accesibility: some preliminary results. Transportation $5,17-42$.

GEURS, K., \& BERT VAN WEE. (2004). Accessibility evaluation of land-use and transport strategies: review and research directions. Journal of Transport Geography, n/d.

GEURS, K., \& RITSEMA, V. E. (2003). Accessibility evaluation of land-use scenarios: the impact of job competition land-use and infrastructure developments for the Netherlands. Environment and Planning B 30 (1), 68-87.

HANSEN, W. G. (1959). How Accessibility Shapes Land Use. Journal of the American Institute of Planners, 73-76.

HARRIS, B. (2001). Accessibility: Concepts and Applications. Journal of Transportation and Statistics , 4, $\mathrm{n} / \mathrm{d}$.

NIEMEIER, D. (1997). Accessibility: an evaluation using consumer welfare. Transportation 24, 377-396.

SHEEN, Q. (1998). Location characteristics of inner-city neighbourhoods and employment accessibility of low-wage workers. Environment and Planning B 25 (3) , 345-365. 